Braun, N. subglomerata A. Braun e N. translucens (Persoon) C. Agardh emend. R.D. Wood subesp. translucens R.D. Wood var. axilaris (A. Braun) R.D. Wood f. auxilaris. Para cada táxon identificado é apresentada a seguinte informação: referência bibliográfica completa à descrição ou diagnose original, basiômio quando existente, citações na literatura brasileira, descrição minuciosa acompanhada de medidas, pelo menos uma prancha de ilustrações com o total de 122 figuras dos táxons identificados, 13 mapas de distribuição das coletas e das sub-regiões do Pantanal, distribuição geográfica no Brasil a partir da informação em literatura publicada até outubro de 1990 e comentários taxonômicos detalhados incluindose 10 tabelas onde são comparadas as variações métricas dos espécimes examinados com a da literatura especializada, além das comparações morfológicas e métricas entre as espécies Nitella subglomerata A. Braun, N. gollmeriana A. Braun e N. acuminata A. Braun ex Wallman detalhados. Os táxons foram todos identificados a partir de amostras populacionais. Foram elaboradas chaves artificiais para os gêneros, espécies, variedades e formas taxonômicas, de acordo com as características vegetativas e reprodutivas. Os táxons que apresentaram maior número de coletas para as subregiões do Pantanal foram, respectivamente: Chara guirensis R. Bicudo com $80 \%$, C. rusbyana Howe com $58 \%$, Nitella furcata (Roxburgh ex Bruzelius) C. Agardh emend. R.D. Wood subesp. furcata var. sieberi (A. Braun) R.D. Wood f. microcarpa (A. Braun) R.D. Wood com 50\% e N. furcata (Roxbugh ex Bruzelius) C. Agardh emend. R.D. Wood subesp. mucronata (A. Braun) R.D. Wood var. mucronata f. mucronata com $30 \%$ dos táxons coletados. As sub-regiões do Nabileque com $46 \%$, Nhecolandia com 17,6\%, Abobral com 13,9\% e Paraguai com $12,8 \%$, foram as que apresentaram os maiores números de coletas.

\title{
Universidade Federal do Rio de Janeiro/Museu Nacional
}

TíTULO: A família Begoniaceae Bonpl. na Reserva de Macaé de Cima, município de Nova Friburgo, RJ.

AUTORA: Eliane de Lima Jacques

DATA: 6 de janeiro de 1993

LOCAL: $\quad$ Museu Nacional-Universidade Federal do Rio de Janeiro

NÍVEL: Mestrado

BANCA EXAMINADORA: Luiz Emygdio de Mello Filho (orientador) - MN/UFRJ

Margarete Emmerich (presidente) - MN/UFRJ

Lúcia D’Avila Freire de Carvalho - JBRJ

Ariane Luna Peixoto - UFRRJ

RESUMO: O levantamento da família Begoniaceae na Reserva Ecológica de Macaé de Cima, localizada no Município de Nova Friburgo, objetiva contribuir para o conhecimento da flora do Estado do Rio de Janeiro. Os estudos morfológicos e taxinômicos das espécies do gênero Begonia resultaram, para a área, no reconhecimento de 20 espécies, das quais 18 são exclusivamente brasileiras. Constatamos a existência de híbridos naturais na área de estudo. As espécies são reconhecidas de um modo geral, pela forma da lâmina foliar e do fruto. Neste estudo apresentamos chaves de identificação para as espécies, descrição, distribuição geográfica, ambiente onde vivem, dados fenológicos e classificação da categoria conservacionista pelos critérios da Internacional Union for Conservation of Nature and Natural Resources (IUCN).

TíTULO: Estudo morfo-taxonômico de Chaetocarpus Thwaites (Euphorbiaceae Juss.) com especial referência as espécies sul-americanas.

AUTORA: Marccus Vinícius da Silva Alves

DATA: 15 de janeiro de 1993 
LOCAL: $\quad$ Museu Nacional-Universidade Federal do Rio de Janeiro

NÍVEL: Mestrado

BANCA EXAMINADORA: Margarete Emmerich (orientadora) - MN/UFRJ

Luiz Emydgio de Mello Filho - MN/UFRJ

Ariane Luna Peixoto - UFRRJ

Lúcia D’Ávila Freire - JBRJ

Jorge Fontella Pereira - JBRJ

RESUMO: O gênero Chaetocarpus pertence a família Euphorbiaceae Juss., subfamília Acalyphoideae Acherson, tribo Chaetocarpeae (Muell. Arg.) Webster. Apresenta 16 espécies em formações vegetais tropicais dos continentes americano, africano e asiático, sendo que a maior concentração de espécies é observada na região neotropical. Este grupo apresenta inúmeros problemas taxonômicos e morfológicos em virtude principalmente da grande variação fenotípica e da carência de estudos mais detalhados que visem a delimitação mais apurada dos taxa. Estes elementos, são extramamente importantes para a compreensão dos diversos erros observados na identificação de material de herbário e também como explicação para as descrições incompletas. O presente estudo portanto, objetiva contribuir para a elucidação das relações taxonômicas entre os representantes do gênero, com ênfase nas espécies sulamericanas. Para tal, emprega-se informações provinientes de análises da morfologia externa e interna, estudos ecológicos e filogenéticos-biogeográficos.

TÍTULO: Estudo comparativo de duas populações de Stachtarpheta cayennensis (Rich) Vahl., Gervão Roxo.

AUTORA: Débora Omena Futuro

DATA: 12 de fevereiro de 1993

LOCAL Museu Nacional/Universidade Federal do Rio de Janeiro

NÍVEL: Mestrado

BANCA EXAMINADORA: Léa de Jesus Neves (orientadora) - MN/UFRJ

Luiz Emygdio de Mello Filho (presidente) - MN/UFRJ

Berta Lange de Morretes - USP

Maria Auxiliadora C. Kaplan

RESUMO: Foi realizado um estudo comparativo da morfologia, externa e interna do corpo aéreo de Stachtarpheta cayennensis (Rich) Vahl., localizadas no Campus da Universidade Estadual do Rio de Janeiro e em Itaipuaçú - Maricá. Analisaram-se os teores mensais de água, ceras epicuticurales e compostos fenólicos presentes nas folhas, com o intuito de averiguarem-se as correlações existentes entre os fatores ambientais e a concentração destas substâncias. Os resultados apontam para uma grande plasticidade fenotípica da espécie acompanhada por alterações morfológicas, anatômicas e fisiológicas. Dentre as variáveis ambientais avaliadas, a pobreza do solo de Itaipuaçú parece ser fator primordial na microfilia apresentada pela população lá encontrada.

TíTULO: Biologia da reprodução de Sagittaria lancifolia L. subsp. lancifolia (Alismataceae).

AUTORA: Mônica Lopes Pimenta

DATA: 5 de abril de 1993

LOCAL Museu Nacional/Universidade Federal do Rio de Janeiro

NÍVEL: Mestrado

BANCA EXAMINADORA: Wilma Teixeira Ormond (orientadora) - MN/UFRJ

Leda Dau (presidente) - MN/UFRJ 
Maria Célia B. Pinheiro

Luiz Emygdio de Mello Filho - MN/UFRJ

RESUMO: Neste trabalho é apresentado um estudo da biologia da reprodução de Sagittaria lancifolia L. subsp. lancifolia, erva perene, rizomatosa, muito comum no brejo herbáceo da restinga de Maricá. Este estudo inclui uma análise da biologia floral, no sistema de reprodução e dos visitantes das flores. Também são feitas considerações sobre o comportamento fenológico, germinação e importância e implicações das reproduções sexuada e assexuada. O estudo morfológico da inflorescência mostrou que a subsp. lancifolia possui flores femininas, localizadas na parte inferior do eixo principal e dos ramos, e masculinas, na parte mediana e superior. O táxon é monóico, protogínico, floresce e frutifica quase o ano inteiro. Os testes de cruzamento mostraram que a espécie é gueitonogâmica e xenogâmica, sendo que em condições naturais, a gueitonogomia pode ocorrer em até $38 \%$ das inflorescências produzidas. A acentuada assincronia das inflorescências favorece a xenogamia. A reprodução assexuada dá-se através de rizomas, com emissões de membros clonais, que podem afastar-se cerca de $40 \mathrm{~cm}$ da panta-mãe, quando então, separam-se desta.

TÍTULO: Morfologia polínica de Boraginaceae nativas do estado de Santa Catarina, Brasil.

AUTORA: Rita Scheel

DATA: $\quad 19$ de abril de 1993

LOCAL Museu Nacional/Universidade Federal do Rio de Janeiro

NÍVEL: $\quad$ Mestrado

BANCA EXAMINADORA: Gertrud Monika B. Schatzmayr (orientadora)

Marília da Silva P. Regali

Hiroko Makino Watanabe - IBt/SP

Vânia Gonçalves L. Esteves - MN/UFRJ

Ariane Luna Peixoto - UFRRJ

RESUMO: Com o objetivo de caracterizar a morfologia polínica das Boraginaceae nativas do estado de Santa Catarina, foram estudadas 29 espécies de 6 gêneros uma das quais com duas variedades. Foram definidos nove tipos polínicos a partir do caráter das aberturas e da superfície e em dois casos foi possível uma subdivisão adicional em subtipos considerando diferenças na forma dos grãos e no número de aberturas. Verificou-se que a morfologia polínica corresponde, de modo geral, ao agrupamento taxinômico das espécies exceto numa exceção do gênero Heliotropium para a qual é sugerida uma reavaliação da taxonomia.

TítULO: Cactaceae da área de proteção da Massambaba. Rio de Janeiro, Brasil.

AUTORA: Maria de Fátima Freitas

DATA: $\quad 6$ de maio de 1993

LOCAL Museu Nacional/Universidade Federal do Rio de Janeiro

NÍVEL: Mestrado

BANCA EXAMINADORA: Jorge Fontella Pereira (orientador) - JBRJ

Ariane Luna Peixoto (presidente) - UFRRJ

Dorothy Sue Dunn de Araujo - FEEMA-RJ

Lúcia D'Ávila Freire de Carvalho - JBRJ

RESUMO: Este trabalho consta de um levantamento das espécies de Cactaceae encontradas na planície arenosa da Área de Proteção Ambiental da Massanbaba (APA-Massanbaba), que ocupa parte dos Municípios de Saquarema, Araruama e Arraial do Cabo, no Estado do Rio de Janeiro. Baseado nos estudos 
do material coletado para este trabalho, foram reconhecidos os seguintes gêneros e espécies para a área de estudo: Pereskia Mill.: P. aculeata Mill.; Opuntia Mill.: O. brasiliensis (Willd.) Haw., O. monacantha (Willd.) Haw,; Cereus Mill.: C. fernambucensis Lem.; Melocactus Link \& Otto: M. violaceus Pfeiff. subsp. violaceus; Pilosocereus (Lem.) Byles \& Rowley: P. arrabidae (Lem.) Byles \& Rowley; Epiphlyllum Haw.: E. phyllanthus (L.) Haw. var. phyllanthus; Hylocereus Britton \& Britton \& Rose: H. undatus (L.) Britton \& Rose; Selenicereus (Berger) Britton \& Rose: S. setaceus (Salm-Dyck ex DC) Werderman; Lepismium Pfeiff.: L. cruciforme (Vell.) Miq.; Rhipsalis Gaertn: R. crispata (Haw.) Pfeiff., R. oblonga Loefgren e $R$. pachyptera Pfeiff. São apresentados chave analítica para as espécies, descrições, comentários, observações de campo, ilustrações, fenologia e distribuição das espécies nas zonas de vegetação da região em estudo.

TíTULO: Alchornea Sw. sec. Alchornea (Euphorbiaceae), espécies ocorrentes no estado do Rio de Janeiro.

AUTORA: Ivete Maria da Silva

DATA: 6 de agosto de 1993

LOCAL Museu Nacional/Universidade Federal do Rio de Janeiro

NÍVEL: Mestrado

BANCA EXAMINADORA: Arline de Souza de Oliveira (orientador) - MN/UFRJ

Luiz Emygdio de Mello Filho (presidente) - MN/UFRJ

Ariane Luna Peixoto - UFRRJ

Luci de Senna Valle

RESUMO: O presente trabalho realiza o estudo taxinômico das espécies de Alchornea Sw. sec. Alchornea ocorrentes no estado do Rio de Janeiro e compreende o tratamento de Alchornea triplinervia (Spreng.) Müll. Arg. var. triplinervia (Spreng.) Müll. Arg. var. janeirensis (Casar.) Müll. Arg., Alchornea iricurana Casar. e Alchornea sidaefolia Müll. Arg. Estes taxa, no Brasil, ocorrem desde o Ceará até o sul do país e encontram-se nas planícies costeiras, nas regiões de encostas e em matas de altitudes mais elevadas. Possuem uma enorme quantidade de nomes populares, sendo os mais predominantes Tanheiro, "tapiá", "tapiá-graçú" e "iricurana". São plantas em geral diócas de porte arbóreo, com exceção de Alchornea triplinervia var. janeirensis que se representa por arvoretas ou arbustos. As folhas dessas espécies e variedades são caracteristicamente 3-nervadas e revestidas de tricomas estrelados que se repetem nas demais partes da planta. Em Alchornea sidaefolia mostram-se estrelado-adpressos e estrelado-fasciculados e suas folhas, devido ao indumento, são nitidamente discolores. Alchornea iricurana pode também * apresentar, nos bordos foliares, tricomas simples, unicelulares e geralmente caducos.

TítUlO: Estudos taxonômicos do gênero Bathysa Presl (Rubiaceae, Rondeletiae) no Brasil.

AUTOR: Pedro Germano Filho

DATA: $\quad 17$ de agosto de 1993

LOCAL Museu Nacional/Universidade Federal do Rio de Janeiro

NÍVEL: $\quad$ Mestrado

BANCA EXAMINADORA: Ariane Luna Peixoto (orientadora) - UFRRJ

Luiz Emygdio de Mello Filho - MN/UFRJ

Josafá Carlos Siqueira - UC

Graziela Maciel Barroso - JBRJ

RESUMO: O gênero Bathysa Presl engloba 13 espécies de árvores, arvoretas ou arbustos ocorrentes no Panamá, Venezuela, Guiana Francesa, Peru, Bolívia e Brasil. No Brasil, ocorrem 7 espécies todas 
exclusivas da Mata Atlântica das Regiōes Sul e Sudeste: B. mendoncea, B. gymnocarpa, B. sylvestre, B. australis, B. stipulate, B. nichdsonii e B. cuspidata. O estudo taxonômico envolveu a análise das coleções dos principais herbários brasileiros, de tipos e fotografias de tipos, bem como material vivo coletado em expedições de campo. São apresentados para cada espécie mapas de distribuição geográfica, dados de tecnologia, status da conservação e comentários.

TítUlo: $\quad$ Faramea Aublet (Rubiaceae): estudo taxonômico das espécies da seção Homaloclados (Hooker) Müller Argovensis.

AUTOR: $\quad$ Mario Gomes

DATA: 20 de agosto de 1993

LOCAL Museu Nacional/Universidade Federal do Rio de Janeiro

NÍVEL: Mestrado

BANCA EXAMINADORA: Graziela Maciel Barroso (orientadora) - JBRJ

Ariane Luna Peixoto (presidente) - UFRRJ

Jorge Fontella Pereira - JBRJ

Josafá Carlos de Siqueira - UC

RESUMO: O presente trabalho trata do estudo taxonômico das quatorze espécies da Seção Homaloclados (Hook.) Müll. Arg., distribuídas nos estados de Minas Gerais, Espírito Santo e Rio de Janeiro, que são aqui redescritas e ilustradas, reavaliadas suas características taxonômicas e rearranjadas, seguindo uma ordem evolutiva, de acordo com critérios estabelecidos e ordenadas em chave analítica, possibilitando a sua identificação. É apresentada uma tabela com dados fenológicos e fornecidas informações sobre os diversos ambientes onde medram essas espécies, bem como uma lista dos nomes dos coletores e mapas de distribuição geográfica.

TíTULO: Contribuição ao conhecimento das espécies brasileiras do gênero Stilpnopappus Mart. ex DC. (Compositae-Vernonieae) - Seção Stilpnopappus.

AUTOR: Roberto Lourenço Esteves

DATA: 20 de agosto de 1993

LOCAL Museu Nacional/Universidade Federal do Rio de Janeiro

NÍVEL: Mestrado

BANCA EXAMINADORA: Graziela Maciel Barroso (orientadora) - JBRJ

Luiz Emygdio de Mello Filho - MN/UFRJ

Lúcia D'Ávilla Freire de Carvalho - JBRJ

Josafá Carlos de Siqueira - UC

RESUMO: O gênero Stilpnopappus Mart. ex DC. (Compositae-Vernonieae) está representado por três seções: Strophopappus, Xiphochaeta e Stilpnopappus. Neste trabalho apresenta-se uma revisão taxinômica da seção Stilpnopappus. Esta é composta por oito espécies sendo praticamente endêmica da região nordeste, ocorrendo em áreas onde predominam os seguintes tipos de vegetação: caatinga, campo ruprestre, cerrado e restinga. No estudo taxinômico da seção, apresenta-se uma chave para identificar suas espécies, acompanhada de descrições, distribuição geográfica, comentários e ilustrações, incluindo desenhos e fotografias dos tipos. Uma nova entidade é descrita: S. semirianus. Três novos sinônimos são propostos: S. procumbens sob S. trichospiroides, S. pickelii sob S. pratensis e S. pratensis var. crotonifolia sob S. pratensis var. pratensis.

TítUlo: $\quad$ Espécies brasileiras de Eriotheca Schott \& Endlicher (Bombacaceae). 
AUTORA: Vera Lúcia Campos Martins

DATA: 25 de agosto de 1993

LOCAL Museu Nacional/Universidade Federal do Rio de Janeiro

NÍVEL: Mestrado

BANCA EXAMINADORA: Ariane Luna Peixoto (orientadora) - UFRRJ

Luiz Emygdio de Mello Filho - MN/UFRJ

Jorge Fontella Pereira - JBRJ

Graziela Maciel Barroso - JBRJ

RESUMO: Apresenta-se neste trabalho uma revisão taxonômica do gênero Eriotheca Schoot \& Endl. (Bombacaceae) o qual está constituído por 2 subgêneros: Macrosiphon, compreendendo 2 espécies, ocorrentes na Hiléia Amazônica e Eriotheca, com 11 espécies, 3 subespécies e 2 variedades. Trata-se de um gênero neotropical, com ocorrência na América do Sul. No tratamento taxonômico do gênero, apresenta-se uma chave dicotômica para a identificação dos seus taxa, acompanhados de descrição, comentários, distribuição geográfica, habitat, etimologia, fenologia, nomes vulgares, ilustrações e tabelas.

TÍTULO: As espécies brasileiras de Neptunia Lour. (Leguminosae - Mimosoideae).

AUTORA: Jorge Gonzaga Filho

DATA: $\quad 13$ de setembro de 1993

LOCAL Museu Nacional/Universidade Federal do Rio de Janeiro

NÍVEL: Mestrado

BANCA EXAMINADORA: Luiz Emygdio de M. Filho (orientador) - MN/UFRJ

Margarete Emmerich (presidente) - MN/UFRJ

Ariane Luna Peixoto - UFRRJ

Jorge Pedro P. Carauta - FEEMA-RJ

RESUMO: O gênero Neptunia Lour. (Leguminosae, Mimosoidae) encontra-se representado no Brasil por duas espécies: Neptunia oleracea e N. plena, ambas presentes nas regiōes Norte e Nordeste e, menos comumente, na Região Sul. Este trabalho visa atualizar os dados existentes acerca dessas duas espécies. Foram consultados herbários e toda a bibliografia referente às espécies e ao gênero, permitindo a elaboração de descrições e o posicionamento taxonômico de cada planta. São fornecidas figuras dos principais caracteres de flores e frutos, além da comparação entre os folíolos e epiderme, estabelecendo-se a diferenciação dos taxa. Também é apresentada uma chave analítica para as espécies em estudo, mapas de distribuição geográfica, listagem de locais onde ocorrem, dados acerca da ecologia e usos.

TíTULO: Graminea (Poaceae) da área de proteção ambiental da Massambaba, municípios de Saquarema a Arraial do Cabo - Rio de Janeiro, Brasil.

AUTOR: Luiz Sergio Pereira Sarahyba

DATA: 17 de novembro de 1993

LOCAL Museu Nacional/Universidade Federal do Rio de Janeiro

NÍVEL: Mestrado

BANCA EXAMINADORA: Margarete Emmerich (orientadora) - MN/UFRJ

Dorothy Sue Dunn de Araújo (presidente) - FEEMA-RJ

Ariane Luna Peixoto - UFRRJ

Tatiana Sendulsky - IBt/SP

RESUMO: Levantamento das espécies de Gramíneas que ocorrem na Área de Proteção Ambiental da 
Massambaba na qual estão incluídas as Reservas Ecológicas Estaduais de Jacarepiá e da Massambaba, por representarem as áreas mais bem conservadas daquela restinga que se estende do Município de Saquarema ao de Arraial do Cabo. Os resultados apresentados consistem em uma chave de identificação, descrição e ilustração das 25 espécies, informações sobre a distribuição geográfica, bem como comentários sobre a ocorrência das mesmas nas diferentes comunidades vegetais da restinga. Os gêneros são os seguintes: Andropogon (2 sp.), Axonopus (1 sp.), Cenchrus (1 sp.), Chloris (1 sp.), Colanthelia (1 sp.), Cryptochloa (1 sp.), Dactyloctenium (1 sp.), Ligitaria (1 sp.), Eragrostis (1 sp.), Eriochrysis (1 sp.), Ichnanthus (1 sp.), Lasiacis (1 sp.), Panicum (2 sp.), Paspalum (4 sp.), Pharus (1 sp.), Paddia (1 sp.), Rhynchelytrum (1 sp.), Saccharum (1 sp.), Sporolobus (1 sp.), Stenotaphrum (1 sp.). Dois perfis topográficos registram a ocorrência das espécies e as diferentes comunidades vegetais. Colanthelia cingulata é referida como nova ocorrência para o estado do Rio de Janeiro.

TítUlO: Malpighiaceae da Reserva Ecológica de Macaé de Cima, Nova Friburgo, Rio de Janeiro, Brasil.

AUTOR: André Marcio de Araujo Amorim

DATA: 19 de novembro de 1993

LOCAL Museu Nacional/Universidade Federal do Rio de Janeiro

NÍVEL: Mestrado

BANCA EXAMINADORA: Jorge Fontella Pereira (orientador)- JBRJ

Lúcia D’ Ávila Freire de Carvalho (presidente) - JBRJ

Maria Candida Henrique Mamede -IBt/SP

Ariane Luna Peixoto - UFRRJ

RESUMO: Apresenta-se o levantamento taxonômico das espécies de Malpighiaceae encontradas na Reserva Ecológica de Macaé de Cima no município de Nova Friburgo, RJ, como contribuição ao conhecimento da flora da mata atlântica do Brasil. Com base em estudo do material coletado para este trabalho, observações das populações no campo e consultas de herbários brasileiros são reconhecidos os seguintes táxons para a região estudada: Banisteriopsis membranifolia (Adr. Juss.) Gates; Byrsonima laevigata (Poir.) DC., B. laxiflora Griseb., B. myricifolia Griseb.; Heteropteris anomala Adr. Juss. var. anomala, H. leschenaultiana Adr. Juss., H. nitida (Lam.) Kunth., H. sericea (Cav.) Adr. Juss. var. sericea; Hiraea gaudichaudiana (Adr. Juss.); Stigmaphyllon gayanum Adr. Juss., Stigmaphyllon sp.; Tetrapterys crebriflora Adr. Juss., T. ialandiana Adr. Juss. e T. lucida Adr. Juss. No tratamento taxonômico, apresentam-se chaves analíticas para a identificação das espécies, seguindo-se descrições e comentários acerca de aspectos morfológicos, fenológicos e de distribuição geográfica das mesmas, com ilustrações e mapas. Assinalam-se cinco novos sinônimos, bem como, pela primeira vez a ocorrência de Byrsonima laevigata no estado do Rio de Janeiro.

TítUlO: Vrisea Lindley (Bromeliaceae) na Reserva Ecológica de Macaé de Cima, Nova Friburgo, Rio de Janeiro, Brasil.

AUTORA: Andréa Ferreira da Costa

DATA: $\quad 16$ de dezembro de 1993

LOCAL Museu Nacional/Universidade Federal do Rio de Janeiro

NÍVEL: Mestrado

BANCA EXAMINADORA: Jorge Fontella Pereira (orientador) - JBRJ

Ariane Luna Peixoto (presidente) - UFRRJ

Maria das Graças Lapa Wanderley - IBt/SP

Lúcia D'Ávila Freire - JBRJ 
RESUMO: Este trabalho consta do estudo taxonômico do gênero Vrisea Lindley na Reserva Ecológica de Macaé de Cima, Nova Friburgo, estado do Rio de Janeiro, Brasil. Foram estudadas 14 espécies: V. altomacaensis Costa, V. arachonoides Costa, V. bituminosa Wawra var.bituminosa, V. carinata Wawra var. carinata, V. haematina L.B. Smith, V. heterostachys (Baker) L.B. Smith, V. hieroglyphica (Carriere) E. Morren var. hieroglyphica, V. hydrophora Ule, V. inflata (Wawra) Wawra, V. Iongicaulis (Baker) Mez. V. longiscapa Ule., V. paraibica Wawra, V. sparsiflora L.B. Smith e V. vagans (L.B. Smith) L.B. Smith, sendo as duas primeiras novas para a ciência. Foi sinonimizada à variedade típica $V$. sparsiflora var. breviscapa Pereira. São fornecidos para todas as espécies descrições, sinonímias, estampas, mapas de distribuição geográfica e comentários acerca da morfologia e do comportamento dos táxons em seu ambiente natural.

TítULO: Regeneração de um trecho de "Mata de Restinga" na Reserva Ecológica Estadual de Jacarepiá, município de Saquarema/RJ.

AUTOR: Cyl Farney C. de Sá

DATA: $\quad 16$ de dezembro de 1993

LOCAL Museu Nacional/Universidade Federal do Rio de Janeiro

NÍVEL: $\quad$ Mestrado

BANCA EXAMINADORA: Graziela Maciel Barroso (orientadora) - JBRJ

Ariane Luna Peixoto (presidente) - UFRRJ

Paulo Yoshio Kageyama - USP

Rogério Ribeiro Oliveira

RESUMO: Um trecho de Floresta de Restinga, na Reserva Ecológica Estadual de Jacarepiá, Saquarema/ RJ, foi estudado após 6 anos de sucessão secundária. A área foi perturbada por tratores em 1986 para abrir arruamentos de um empreendimento imobiliário, tendo permanecida intocada após o embargo e a criação da Reserva. Esta região está submetida as adversidades climáticas oriundas do sistema de Cabo Frio, manisfestada sobretudo no índice pluvimétrico médio de $987 \mathrm{~mm} / \mathrm{ano}$. Os solos são tipicamente pobres. Foram amostradas duas formações que compõem a vegetação perturbada utilizando duas metodologias diferentes: a formação herbáceo/arbustivo foi amostrada pelo Método de Pontos e a formação arbustiva/ arbóreo pelo Método de Parcelas. A composição florística da formação herbáceo/arbustivo é representada por 38 famílias; distribuídas em 78 gêneros e 91 espécies; è na formação arbustivo/ arbórea respectivamente 42 famílias, 85 gêneros e 102 espécies, totalizando 52 famílias, 116 gêneros e 141 espécies para a área como um todo.

\section{Universidade Federal do Rio Grande do Sul}

TITULO: Anatomia foliar de espécies da subtribo Pleurothallidinae (Orchidaceae).

AUTOR: João Luiz Bernardi Ferreira

DATA: $\quad 8$ de junho de 1992

LOCAL: Departamento de Botânica, Universidade Federal do Rio Grande do Sul

NÍVEL: Mestrado

BANCA EXAMINADORA: Paulo Luiz de Oliveira (orientador) - UFRGS

Jorge Ernesto de Araújo Mariath - UFRGS

Alfredo Elio Cocucci - Univ.Córdoba, Argentina

Jane Elizabeth Kraus - USP 